\title{
Perceptions of Popularity-Related Behaviors in the Cyber Context: Relations to Cyber Social Behaviors
}

\author{
Michelle F. Wright \\ Faculty of Social Studies, Masaryk University, 60200 Brno, Czech Republic \\ Correspondence should be addressed to Michelle F. Wright; michelle.wright@mail.muni.cz
}

Received 31 July 2015; Accepted 23 November 2015

Academic Editor: Elena Nicoladis

Copyright ( 2015 Michelle F. Wright. This is an open access article distributed under the Creative Commons Attribution License, which permits unrestricted use, distribution, and reproduction in any medium, provided the original work is properly cited.

\begin{abstract}
Despite acknowledging that adolescents are active users of electronic technology, little is known about their perceptions concerning how such technologies might be used to promote their social standing among their peer group and whether these perceptions relate to their cyber social behaviors (i.e., cyber aggression perpetration, cyber prosocial behavior). To address this gap in the literature, the present study included 857 seventh graders (M age: 12.19; 50.8\% female) from a large Midwestern city in the United States. They completed questionnaires on face-to-face social behaviors, cyber social behaviors, perceived popularity, social preference, and their perceptions of characteristics and activities related to the cyber context which might be used to promote popularity. Findings revealed four activities and characteristics used to improve adolescents' social standing in the peer group, including antisocial behaviors, sociability, prosocial behaviors, and technology access. Using antisocial behaviors in the cyber context to promote popularity was related to cyber aggression perpetration, while controlling for gender, social preference, and perceived popularity. On the other hand, sociability and prosocial behaviors in the cyber context used to improve popularity as well as technology access were associated with cyber prosocial behavior. A call for additional research is made.
\end{abstract}

\section{Introduction}

Early adolescents (ages 11-14) are at an increased risk for perpetrating cyber aggression when compared to children, older adolescents, and young adults [1]. As a result of these research findings, researchers have directed their attention to understanding the risk factors associated with adolescents' perpetration of cyber aggression. Some adolescents engage in aggressive behaviors as a means for attaining and maintaining one's social standing in the peer group [2-4]. Eder [5] defined peer status as adolescents' social standing within their peer group. Social standing in the peer group consists of low levels of peer status (i.e., rejection, unpopularity), average levels of peer status, and higher levels of peer status (i.e., high perceived popularity, high social preference). Considering that adolescents have some of the highest rates of electronic technology usage, it has been proposed that they might utilize these technologies to achieve a higher social standing in the peer group [6]. Few studies have empirically tested this proposal, but few have found that high perceived popularity is related to cyber aggression perpetration, while high social preference was associated with cyber prosocial behavior [7]. Despite these preliminary findings, little attention has focused on the cyber context as a tool to advance adolescents' social standing among their peers. To address this gap in the literature, the aim of the present study was to investigate adolescents' perceptions of the different ways that the cyber context can be used to become more popular among their peer group at school and whether these perceptions relate to their engagement in cyber social behaviors (i.e., cyber aggression, cyber prosocial behavior). Because perceived popularity and social preference relate to cyber social behaviors, this study also accounted for these attained statuses to more thoroughly focus on how adolescents' perceptions of popularity-motivated activities in the cyber context relate to cyber social behaviors. This procedure will help provide a better understanding of these associations beyond the impact of attained status on adolescents' social behaviors. 


\section{Popularity and Social Behaviors}

Research has revealed that there are two distinctive subtypes of popularity in adolescents' peer groups: perceived popularity and social preference $[2,3]$. Popularity is the term used to describe both perceived popularity and social preference, though both of these subtypes relate to different behavioral characteristics. As a reputational type of popularity, perceived popularity does not necessarily reflect adolescents' likeability among peers [4]. This form of popularity is characterized by high social prestige and social centrality within the peer group. Adolescents who are perceived as popular might not necessarily be well-liked by their peers, although they are emulated. On the other hand, social preference is characterized by social acceptance and adolescents with high levels of social preference are generally well-liked by their peers [2]. The type of social behaviors popular adolescents engage in depends on the subtype of popularity they exhibit. In particular, adolescents with high levels of perceived popularity engage in relational aggression (e.g., harming another individual by damaging their relationships or peer status, including rumor spreading, gossiping, relationship manipulation, and social exclusion) [8]. In contrast, adolescents with high levels of social preference do not perpetrate relational aggression, and instead they are more likely to engage in prosocial behavior (e.g., behaviors that involve a concern for the welfare of others and include behaviors such as helping one's peers and cheering peers up when the peer needs it) $[3,9]$. Perceived popularity is inconsistently related to adolescents' engagement in prosocial behavior. Some research has revealed that these adolescents are bistrategic, engaging in both relational aggression and prosocial behavior. However, not all research indicates that perceived as popular adolescents utilize prosocial behavior.

Adolescents have fully embraced electronic technology, with $92 \%$ going online once a day [10]. Although various scholars recognize the importance of electronic technologies in adolescents' lives, little attention has been given to whether adolescents might utilize the cyber context to attain or maintain their social standing among their peers [11]. This research is especially important as the desire for a higher peer status increases in adolescence $[4,12]$. Therefore, the cyber context might be used as another tool for gaining peer status in adolescence. Some research has focused on the linkages of adolescents' popularity to their social behaviors in the cyber context. In this research, Schoffstall and Cohen [13] and Wright [7] found that perceived popularity was related positively to cyber aggression perpetration. In addition, Wright [7] found a negative relationship between cyber aggression perpetration and social preference. Her results also revealed that social preference is related positively to cyber prosocial behavior, but cyber prosocial behavior was not associated with perceived popularity. These handful of studies provide a firm foundation for understanding the relationships between popularity types and cyber social behaviors. However, little is known about how adolescents utilize electronic technologies and the cyber context to maintain or promote their popularity within their peer group. In the face-to-face context, research findings link children's and adolescents' perceptions of what makes a girl or a boy popular to their aggressive behaviors. In particular, Xie et al. [12] found that children and adolescents who believed that deviance contributed to popularity had higher ratings of aggression as reported by teachers and peers. As a result of these findings, combined with adolescents' increasing usage of electronic technology, it might be reasonable to propose that adolescents' perceptions of their popularity-motivated activities in the cyber context that promote a higher social standing could contribute to their cyber social behaviors.

\section{The Present Study}

Considering that many adolescents have fully embraced electronic technologies, it might be likely that they perceive these technologies as serving a variety of purposes. One such perception is that these technologies might be used to increase popularity among the peer group, which might contribute to adolescents' social behaviors in the cyber context. This might be a reasonable expectation as popularity is a major concern among adolescents, resulting in distinctive behavioral patterns. Therefore, there was one major goal of the present study. This goal was to investigate adolescents' perceptions of different activities in the cyber context that are used to increase their popularity and how such activities might relate to their cyber social behaviors. Gender, attained status (i.e., perceived popularity, social preference), and faceto-face social behaviors were included as control variables. Controlling for gender is important as it is an inconsistent predictor of cyber aggression [14-17]. It is unknown whether gender might relate to cyber prosocial behavior, but, in the face-to-face context, this behavior is typically associated with girls [18]. Including perceived popularity and social preference as control variables is important as these subtypes of popularity are consistently associated with differential behavioral profiles, allowing for the ability to examine the associations investigated in this study beyond the effect of these statuses [2-5]. Furthermore, controlling for face-toface social behaviors allows for a better understanding of the relationships examined in this study, especially since face-to-face social behaviors and cyber social behaviors are correlated.

\section{Method}

4.1. Participants. Participants for this study were 857 seventh graders ( $\mathrm{M}$ age: $12.19 ; 50.8 \%$ female) from seven middle schools from the suburbs of a large Midwestern city. Adolescents primarily self-identified as White (71.1\%). Middle schools were located in predominantly middle class neighborhoods, and between $10 \%$ and $25 \%$ of students from the schools received free or reduced lunch.

4.2. Procedures. To recruit schools for this study, a list of over 200 middle schools in the suburbs of a large Midwestern city was created. Of these schools, 10 were randomly selected to receive an email invitation, which was directed to the school principals. The email described the nature of the study, what adolescents would be expected to do, how adolescents 
could participate, and how important it was for adolescents to participate in the study. Seven school principals responded to the email and they requested a meeting among themselves, teachers, and the principal investigator of the study. During this meeting, the principal investigator explained the purpose of the study and described the questionnaires that adolescents would be expected to complete if they were to participate. After this meeting, classroom announcements were made, which reiterated the information from the principal and teacher meetings. Next, 961 letters and parental permission slips were sent home with adolescents. There were 857 permission slips returned with consent, 40 without consent, and 64 that were never returned to school.

Data collection took place over six weeks. Adolescents provided their assent before receiving the questionnaires. All adolescents provided their assent. They completed demographic information (e.g., age, gender, and ethnicity), selfreported cyber social behaviors perpetration, self-reported face-to-face social behaviors perpetration, peer nominations of perceived popularity and social preference, and what makes someone popular with their peers (i.e., Popularity Perceptions Measure).

\subsection{Measures}

4.3.1. Self-Reported Cyber Aggressive and Prosocial Behaviors. There were thirteen items included in this questionnaire, which were rated on a scale of 1 (not at all) to 5 (all of the time) [19]. The items asked adolescents to rate how often they engaged in cyber aggression (nine items; e.g., spread untrue and bad rumors about another peer online or through text messages; $\alpha=.89$ ) and cyber prosocial behavior (four items; e.g., cheer other peers up online or through text messages; $\alpha=.87$ ).

4.3.2. Self-Reported Face-to-Face Aggressive and Prosocial Behaviors. Similar to the self-reported cyber social behaviors perpetration questionnaire, this questionnaire included thirteen items in which adolescents were asked to rate how often they engaged in face-to-face aggression (nine items; e.g., spread untrue and bad rumors about another peer) and faceto-face prosocial behavior (four items; e.g., cheer other peers up online or through text messages) [17]. The items were rated on a scale of 1 (not at all) to 5 (all of the time). Cronbach's alphas were .86 for face-to-face aggression and .87 for faceto-face prosocial behavior.

\subsubsection{Peer Nominations of Perceived Popularity and Social} Preference. This questionnaire included four items, which asked adolescents to nominate peers whom they believed fit the descriptions described in the items. Two items (i.e., liked the most, liked the least) were used for social preference [2]. Similarly, two items (i.e., who were popular, who were unpopular) were used for perceived popularity $[3,4]$. After data was collected, the total number of nominations was tallied for each participant and then standardized according to class and school. Standardization accounts for unequal school and classroom sizes [2]. The standardized "like least" item was subtracted from the standardized "like most" item, resulting in one score for social preference, with higher scores suggesting greater social preference. Likewise, the "unpopular" item was subtracted from the "popular" item, yielding a final score on perceived popularity, with higher scores indicating greater perceived popularity.

4.3.4. Popularity Perceptions Measure. This measure listed various characteristics and activities that occurred in the cyber context to make someone popular with their peers at school [6]. There were 21 items included in this questionnaire, and these were rated on a scale of 1 (very unpopular) to 5 (very popular). Before the items, adolescents read a description which indicated that the behaviors described on the questionnaire were used to become more popular among their peers. The questionnaire included four subscales, including sociability (six items; e.g., receiving a lot of text messages), antisociality (six items; e.g., calling peers mean names online or through text messages), prosociality (four items; e.g., helping peers out online or through text messages), and technology accessibility (five items; e.g., having access to all the latest electronic technologies). Cronbach's alphas were acceptable $(\alpha=.89$ for sociability, $\alpha=.90$ for antisociality, $\alpha=.90$ for prosociality, and $\alpha=.86$ for technology accessibility).

\section{Results}

Confirmatory factor analysis was performed before examining the hypothesized relationships using a structural regression model. The model demonstrated adequate fit indices, $\chi^{2}=961.56, \mathrm{df}=676, p<.001, \mathrm{CFI}=.98, \mathrm{TLI}=.97$, RMSEA $=.05$, SRMR $=.05$. All standardized factor loadings were significant $(p<.001)$ and had good magnitudes. Therefore, the items were used as indicators of the latent variables for the structural regression model.

A correlation matrix was created for all variables included in the study using the scaled scores (see Table 1). The findings revealed that cyber aggression perpetration was associated positively with face-to-face aggression, perceived popularity, and the sociability and antisociality subscales. On the other hand, cyber aggression perpetration was related negatively to social preference. Face-to-face aggression was related positively to perceived popularity and the antisocial behavior subscale, while it was associated negatively with social preference and the prosocial behavior subscale. Cyber prosocial behavior was associated positively with face-to-face prosocial behavior, social preference, sociability, prosociality, and technology accessibility subscales. Face-to-face prosocial behavior was related to the social preference and the subscales of sociability, prosocial behavior, and technology access. In addition, face-to-face and cyber prosocial behaviors were negatively linked to the antisociality subscale. The sociability subscale was related positively to the prosocial behavior subscale and social preference. Furthermore, the antisocial behavior subscale was linked positively to perceived popularity, though it was related negatively to the prosocial behavior subscale and social preference. Perceived popularity and social preference were associated positively with each other. 
TABLE 1: Correlations among all variables.

\begin{tabular}{|c|c|c|c|c|c|c|c|c|c|c|}
\hline & 1 & 2 & 3 & 4 & 5 & 6 & 7 & 8 & 9 & 10 \\
\hline (1) CAP & - & & & & & & & & & \\
\hline (2) $\mathrm{CPB}$ & -.09 & - & & & & & & & & \\
\hline (3) FAP & $.61^{* * *}$ & -.08 & - & & & & & & & \\
\hline (4) FPB & -.01 & $.33^{* * *}$ & -.11 & - & & & & & & \\
\hline (5) Sociability subscale & $.19^{*}$ & $.23^{* *}$ & .11 & $.28^{* * *}$ & - & & & & & \\
\hline (6) Antisocial behavior subscale & $.31^{* * *}$ & $-.21^{* *}$ & $.23^{* *}$ & $-.23^{* *}$ & .13 & - & & & & \\
\hline (7) Prosocial behavior subscale & .01 & $.26^{* * *}$ & $-.19^{*}$ & $.22^{* *}$ & $.28^{* * *}$ & $-.21^{*}$ & - & & & \\
\hline (8) Technology accessibility subscale & .08 & $.18^{*}$ & .03 & $.18^{*}$ & .14 & .03 & .02 & - & & \\
\hline (9) Perceived popularity & $.32^{* * *}$ & .03 & $.34^{* * *}$ & .08 & .12 & $.27^{* * *}$ & .07 & .04 & - & \\
\hline (10) Social preference & $-.29^{* * *}$ & $.33^{* * *}$ & $-.32^{* * *}$ & $.30^{* * *}$ & $.18^{*}$ & $-.30^{* * *}$ & $.20^{*}$ & $.18^{*}$ & $.18^{*}$ & - \\
\hline
\end{tabular}

Note. CAP: cyber aggression perpetration; CPB: cyber prosocial behavior; FAP: face-to-face aggression perpetration; FPB: face-to-face prosocial behavior. ${ }^{*} p<.05 .{ }^{* *} p<.01 .{ }^{* * *} p<.001$.

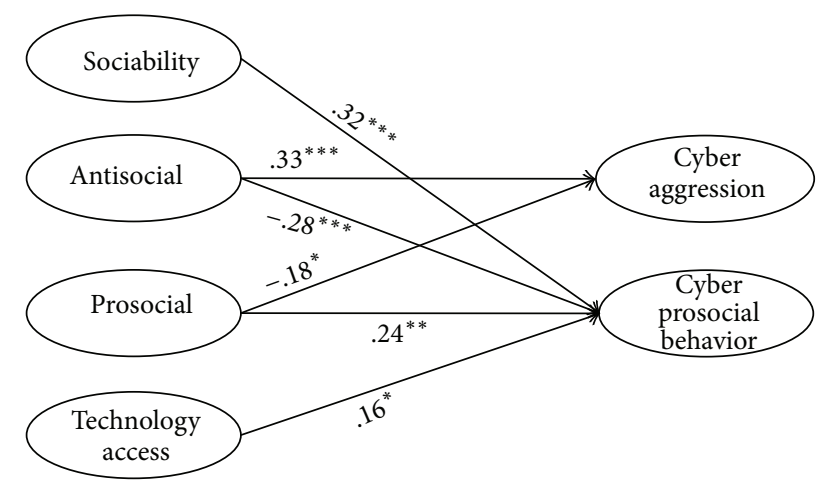

Figure 1: Structural regression model. Only significant paths are displayed. Control variables of perceived popularity, face-to-face social behaviors, social preference, and gender are also not shown. Gender was not related to any of the variables in the structural regression model. Social preference was related positively to the prosocial factor $(\beta=.24, p<.001)$ and cyber prosocial behavior $(\beta=.36, p<.001)$, while perceived popularity was associated positively with the antisocial factor $(\beta=.19, p<.05)$ and cyber aggression perpetration $(\beta=.41, p<.001) .{ }^{*} p<.05 .{ }^{* *} p<.01$. ${ }^{* * *} p<.001$.

Structural regression was used to model direct paths from the popularity perceptions subscales to cyber aggression perpetration and cyber prosocial behavior. Paths were added from face-to-face social behaviors to cyber social behaviors (i.e., aggression, prosocial behavior). Gender, social preference, and perceived popularity were allowed to predict all variables in the model by specifying direct paths from these variables to all the others. The structural model fits the data well, $\chi^{2}=1123.03, \mathrm{df}=701, p<.001$, CFI $=$ .99 , TLI $=.98$, RMSEA $=.03$, SRMR $=.04($ see Figure 1$)$. After controlling for gender, perceived popularity, social preference, and face-to-face social behaviors (i.e., aggression, prosocial behavior), antisociality was related positively to cyber aggression perpetration $(\beta=.33, p<.001)$, but it was associated negatively with cyber prosocial behavior $(\beta=-.28, p<.001)$. Prosociality was negatively linked with cyber aggression perpetration $(\beta=-.18, p<.05)$. In addition, sociability $(\beta=.32, p<.001)$, prosociality $(\beta=.24$, $p<.01)$, and technology accessibility $(\beta=.16, p<.05)$ were each associated positively with cyber prosocial behavior. Gender was not related to any variable in the study. Social preference was related positively to the prosocial behavior factor $(\beta=.24, p<.001)$ and cyber prosocial behavior $(\beta=$ $.36, p<.001)$, while perceived popularity was associated positively with the antisociality factor $(\beta=.19, p<.05)$ and cyber aggression perpetration $(\beta=.41, p<.001)$.

\section{Discussion}

The aim of the present study was to examine adolescents' perceptions of how various activities and characteristics might promote popularity in their peer group and how adolescents' endorsement of these behaviors and characteristics relate to cyber aggression perpetration and cyber prosocial behavior, while controlling for face-to-face social behaviors, perceived popularity, social preference, and gender. The findings of this study increase our understanding of adolescents' perceptions of using the cyber context to pursue a higher social standing among their peers and how these perceptions relate to their engagement in cyber aggression perpetration and cyber prosocial behavior.

Adolescents' perceptions of using antisocial behaviors in the cyber context to promote one's social standing were associated positively with cyber aggression perpetration, while sociability was unrelated to this behavior. Such findings are intriguing as the sociability and antisociality factors include characteristics that represent social centrality, a characteristic associated with perceived popularity [2-5]. Therefore, the linkage of the antisociality factor to cyber aggression perpetration was not surprising as this factor represents a core feature of perceived popularity, the engagement in aggressive behaviors [4]. The correlational finding regarding sociability is consistent with the characteristics of perceived popularity, although, after including other variables in the model, this factor was unrelated to cyber aggression perpetration. The sociability aspect of promoting one's social standing might also relate to social preference as this subtype of popularity involves peer acceptance. Thus, a potential explanation for the 
lack of relationship between sociability and cyber aggression perpetration in the structural regression model could suggest that other variables, like acting prosocially to promote status, might interfere with this relationship as being accepting of one's peers and prosocial are more tied to social preference. Another proposal is that there might be a cluster of highly popular adolescents who are socially central and emulated, like adolescents with perceived popularity, but that they are also very prosocial, like adolescents with high social preference.

The antisociality factor was related negatively to cyber prosocial behavior, whereas the sociability factor was positively linked with this behavior. Research on the relationship of perceived popularity and prosocial behaviors is mixed, with some researchers finding positive relationships, while others find negative associations $[9,20]$. It might be likely that these findings could be attributed to the different functions of popularity-related behaviors. Thus, adolescents who endorsed the antisocial aspect of perceived popularity might be less inclined to engage in cyber prosocial behaviors, but more likely to engage in cyber aggression. In contrast, adolescents who endorsed the sociability component of popularity might encompass traits associated with social preference as well as perceived popularity, leading them to focus more on the promotion of status using prosocial means. The prosocial factor was related positively to cyber prosocial behavior and not cyber aggression perpetration. This was expected as acting prosocially in the cyber context to promote one's status is more related to social preference [20]. The technology accessibility factor was not associated with cyber aggression perpetration, though it was related positively to cyber prosocial behavior. Interpretation of this finding is difficult. Children and adolescents with greater spending power are likely to be more popular than other children and adolescents who do not [21]. Therefore, having access to the best technology might be a component of popularity, relating to greater prosocial behavior, but not associated with greater cyber aggression.

\section{Limitations and Future Directions}

This study was one of the first to investigate certain activities and characteristics occurring in the cyber context that might promote adolescents' popularity in their peer group and how adolescents' perceptions of these popularity-related activities and characteristics relate to their cyber social behaviors, including cyber aggression and cyber prosocial behavior. Such research is valuable as adolescents frequently utilize electronic technologies and many increasingly desire to promote their social standing in the peer group. This is an important direction in the field. However, there are a few limitations and recommendations to help advance our knowledge of adolescents' popularity-related behaviors and activities in the cyber context. Research utilizing interviews and focus groups should be carried out to understand whether there are more behaviors and characteristics that occur in the cyber context, which adolescents use to promote their social standing in the peer group at school. Furthermore, the research involved a concurrent research design, making it difficult to draw conclusions about the temporal ordering of these relationships and how the various behaviors and characteristics relate to future behaviors. Thus, longitudinal research designs should be carried out to understand the nature and extent of the relationships examined in this study. In addition, this research can also help us understand whether these popularity-related activities and characteristics are associated with increases in adolescents' social standing in the future.

\section{Conclusion}

The present study provided one of the first investigations focused on understanding adolescents' perceptions of utilizing the cyber context to boost their social standing among their peers at school. It was also one of the first studies to understand how adolescents' perceptions of popularityrelated behaviors and characteristics in the cyber context relate to their cyber social behaviors. Research focused on the connections among adolescents, popularity, and the cyber context is valuable as more researchers are beginning to recognize the importance of the media and electronic technology in adolescents' lives, and as a result such tools might serve to promote their peer status. Ultimately, the results help us to understand adolescents' utilization of the cyber context to become more popular and that such pursuit relates to cyber aggression perpetration and cyber prosocial behavior. This research has application to intervention efforts by helping to provide information concerning the identification of adolescents at risk for cyber aggression perpetration. In addition, this research can also help to promote interventions directed at increasing positive interactions in the cyber context.

\section{Conflict of Interests}

The author declares that there is no conflict of interests regarding the publication of this paper.

\section{References}

[1] A. Sevcikova and D. Šmahel, "Online harassment and cyberbullying in the Czech Republic: comparison across age groups," Journal of Psychology, vol. 217, no. 4, pp. 227-229, 2009.

[2] J. D. Coie, K. A. Dodge, and H. Coppotelli, "Dimensions and types of social status: a cross-age perspective," Developmental Psychology, vol. 18, no. 4, pp. 557-570, 1982.

[3] K. M. LaFontana and A. H. N. Cillessen, "Children's perceptions of popular and unpopular peers: a multimethod assessment," Developmental Psychology, vol. 38, no. 5, pp. 635-647, 2002.

[4] A. H. N. Cillessen and L. Mayeux, "From censure to reinforcement: developmental changes in the association between aggression and social status,' Child Development, vol. 75, no. 1, pp. 147-163, 2004.

[5] D. Eder, "The cycle of popularity: interpersonal relations among female adolescents," Sociology of Education, vol. 58, no. 3, pp. 154-165, 1985.

[6] M. F. Wright, "The role of the media and the cyber context in adolescents' popularity," in Living in the Digital Age: SelfPresentation, Networking, Playing, and Participation in Politics, 
P. Lorentz, D. Smahel, M. Metykova, and M. F. Wright, Eds., Muni Press, Brno, Czech Republic, 2015.

[7] M. F. Wright, "Longitudinal investigation of the associations between adolescents' popularity and cyber social behaviors," Journal of School Violence, vol. 13, no. 3, pp. 291-314, 2014.

[8] N. R. Crick and J. K. Grotpeter, "Relational aggression, gender, and social-psychological adjustment," Child Development, vol. 66, no. 3, pp. 710-722, 1995.

[9] M. J. Prinstein and A. H. N. Cillessen, "Forms and functions of adolescent peer aggression associated with high levels of peer status," Merrill-Palmer Quarterly, vol. 49, no. 3, pp. 310-342, 2003.

[10] A. Lenhart, Teens, social media \& technology: overview 2015, http://www.pewinternet.org/2015/04/09/teens-social-mediatechnology-2015/.

[11] D. Smahel, M. F. Wright, and M. Cernikova, "Classification of online problematic situations in the context of youths' development," Communications, vol. 39, no. 3, pp. 233-260, 2014.

[12] H. Xie, Y. Li, S. M. Boucher, B. C. Hutchins, and B. D. Cairns, "What makes a girl (or a boy) popular (or unpopular)? African American children's perceptions and developmental differences," Developmental Psychology, vol. 42, no. 4, pp. 599612, 2006.

[13] C. L. Schoffstall and R. Cohen, "Cyber aggression: the relation between online offenders and offline social competence," Social Development, vol. 20, no. 3, pp. 587-604, 2011.

[14] R. P. Ang and D. H. Goh, "Cyberbullying among adolescents: the role of affective and cognitive empathy, and gender," Child Psychiatry and Human Development, vol. 41, no. 4, pp. 387-397, 2010.

[15] S. Bauman, R. B. Toomey, and J. L. Walker, "Associations among bullying, cyberbullying, and suicide in high school students," Journal of Adolescence, vol. 36, no. 2, pp. 341-350, 2013.

[16] P. Gradinger, D. Strohmeier, and C. Spiel, “Traditional bullying and cyberbullying: identification of risk groups for adjustment problems," Journal of Psychology, vol. 217, no. 4, pp. 205-213, 2009.

[17] M. F. Wright and Y. Li, “The association between cyber victimization and subsequent cyber aggression: the moderating effect of peer rejection," Journal of Youth and Adolescence, vol. 42, no. 5, pp. 662-674, 2013.

[18] N. Eisenberg and P. A. Miller, "The relation of empathy to prosocial and related behaviors," Psychological Bulletin, vol. 101, no. 1, pp. 91-119, 1987.

[19] M. F. Wright, Y. Li, and J. Shi, “Chinese adolescents' social status goals: associations with behaviors and attributions for relational aggression," Youth and Society, vol. 46, no. 4, pp. 566-588, 2014.

[20] J. T. Parkhurst and A. Hopmeyer, "Sociometric popularity and peer-perceived popularity: two distinct dimensions of peer status," Journal of Early Adolescence, vol. 18, no. 2, pp. 125-144, 1998.

[21] P. Adler and P. Adler, Peer Power: Preadolescent Culture and Identity, Rutgers University Press, New Brunswick, NJ, USA, 1998. 

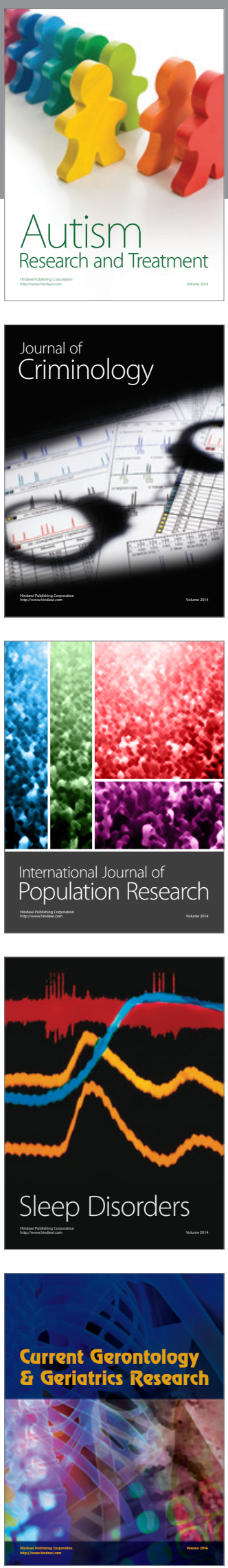
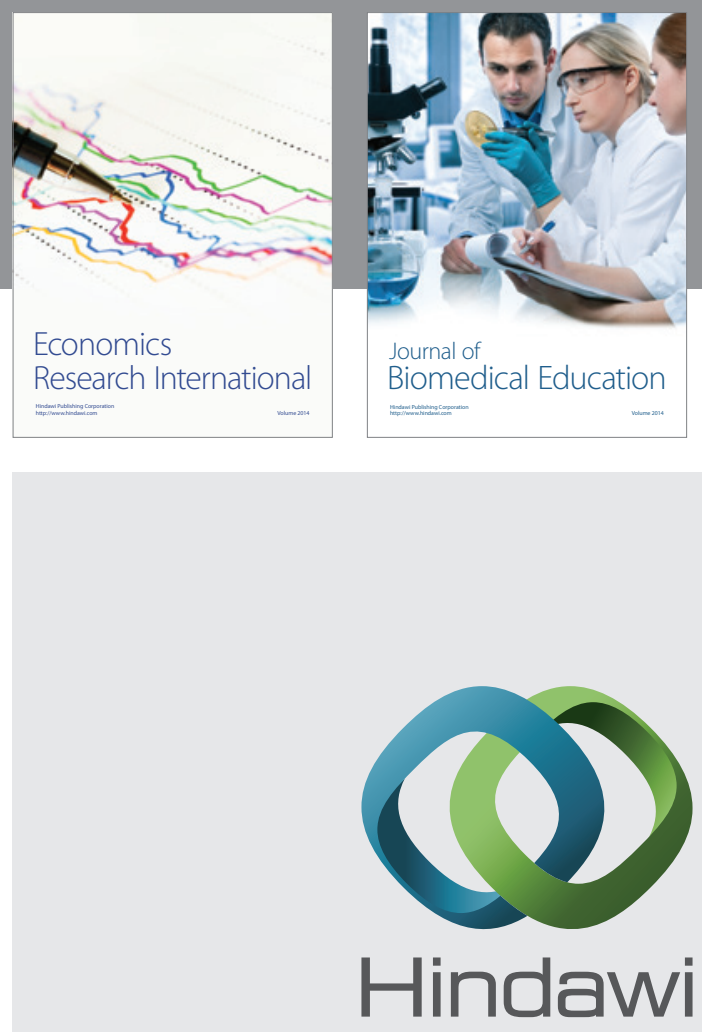

Submit your manuscripts at

http://www.hindawi.com
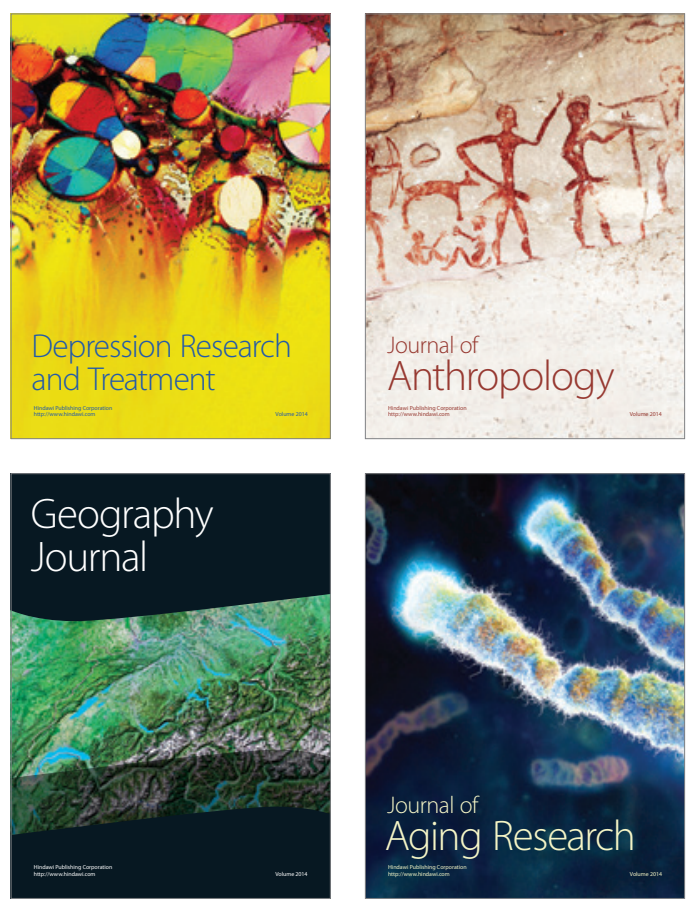
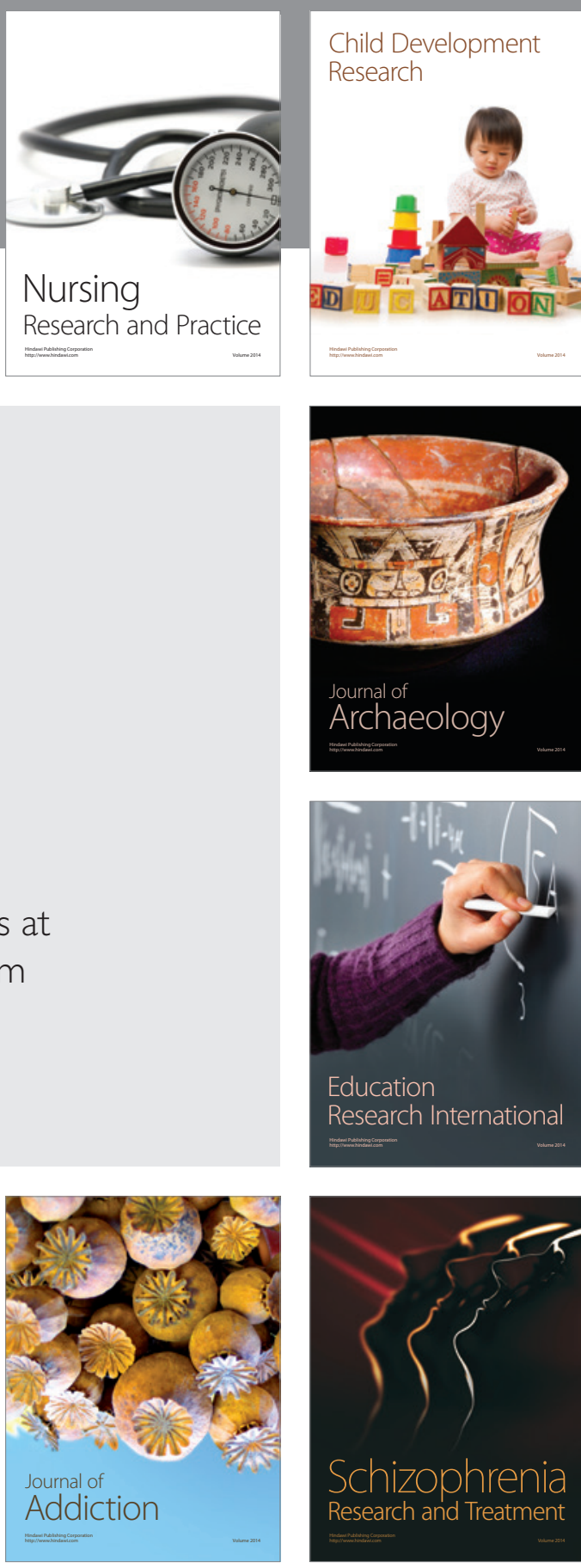

(D)
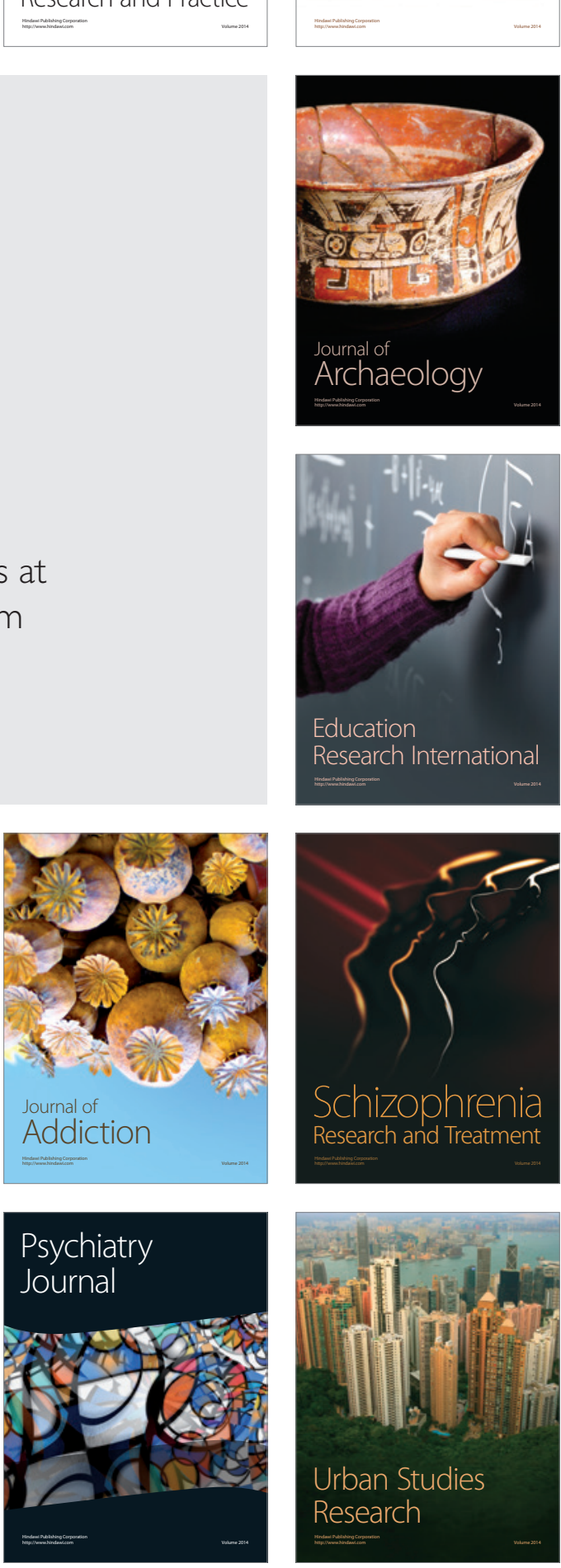